\title{
Douleur et médecine chinoise
}

\section{Pain and Chinese Medicine}

\section{A. Baumelou $\cdot$ B. Liu $\cdot$ J.R. Attali}

Reçu le 5 juin 2015 ; accepté le 8 juin 2015

(C) Lavoisier SAS 2015

« Speakers agreed that, in general, pain is poorly managed, and better strategies are needed. Much emphasis was placed on concerns about opioids, which are widely used for chronic pain but lack compelling evidence for their effectiveness for chronic vs acute pain and have risks and side effects that may exceed benefits when opioids are used chronically. Working group members noted that the evidence of efficacy for complementary approaches for chronic pain is incomplete, and many studies suggest effects are modest; nonetheless, conventional techniques for managing chronic pain, including opioids and surgical and epidural interventions, also have limited efficacy. The balance of risk and benefit suggests that integrative approaches that utilize complementary techniques are promising and deserve more study. "

A. NACCIH, Working Group report. February 2015

La douleur est la première cause de recours aux médecines alternatives et complémentaires [1]. La revue Douleur et Analgésie consacre ce numéro à la prise en charge de la douleur par les techniques de médecine traditionnelle chinoise. L'importance du thème est parfaitement illustrée par une partie des conclusions d'un groupe de travail du National Advisory Council on Complementary and Integrative Health [2] reproduite ci-dessus.

La médecine traditionnelle chinoise est un système de santé qui, s'appuyant sur un cadre philosophique fondamen-

\footnotetext{
A. Baumelou $(\bowtie)$

Centre intégré de médecine chinoise, Université Pierre et Marie Curie Paris 6, groupe hospitalier universitaire Pitié Salpêtrière-Charles Foix

e-mail : alain.baumelou@aphp.fr

B. Liu

Ingénieur de recherche
}

J.R. Attali

Université Paris 13 tal, associe des procédures d'interrogatoire et d'examen clinique, un système diagnostique reposant sur des symptômes, syndromes et des procédures thérapeutiques [3]. En France, l'université Paris 13 a initié un cycle d'enseignement post-universitaire dans les années 1990 et, dès le début, a insisté sur la nécessité d'une connaissance fondamentale des bases de ce système de santé et, pour ce faire, a créé le diplôme d'université Dumétrac, maintenant enseigné à Paris 6 Pierre et Marie Curie.

On retrouvera les bases de l'examen du patient douloureux en médecine chinoise dans une précédente revue [4]. Les bases historiques de la prise en charge de la douleur en médecine chinoise sont reprises dans l'article de C. Despeux dans cette livraison.

La thérapeutique en médecine traditionnelle chinoise associe l'acupuncture, les techniques corps esprit qui regroupent tai qi, qi gong et méditation, le massage tui na et les automassages, les plantes, et la diététique. Ce corpus thérapeutique présente deux caractéristiques (entre autres) par rapport à notre médecine occidentale : il mêle des actions de traitement et de prévention, il n'y a pas de séparation entre le corps et l'esprit.

Actuellement, le traitement par les plantes est la procédure thérapeutique de médecine traditionnelle chinoise la plus répandue en Chine, y compris dans le traitement de la douleur. Il utilise plus de 5000 plantes, mais aussi des produits animaux et des minéraux et la pharmacopée chinoise est probablement la plus riche dans le monde. Néanmoins, les régulations pharmaceutiques européennes ne retiennent qu'une cinquantaine de principes actifs dans leurs monographies. Par ailleurs, les procédures de distribution des plantes dans les pharmacies de médecine traditionnelle (prescription individualisée, multiples associations) sont telles qu'une évaluation rigoureuse est en l'état actuel inenvisageable. Cette valence très importante de la médecine traditionnelle chinoise n'est donc pas abordée dans cette livraison.

La prise en charge de certaines douleurs par l'acupuncture, et par les techniques corps-esprit est largement développée par plusieurs auteurs dans ce numéro. Peut-on juger l'efficacité de cette médecine à l'aune du Discours de la 
Méthode, référence méthodologique de toute notre science médicale conventionnelle ? Peut-on juger l'efficacité de ces traitements avec les instruments de l'evidence based medicine? On connait les résultats de pratiquement toutes les méta-analyses réalisées sur ces procédures : il existe des présomptions d'efficacité mais les patients dans les essais sont trop peu nombreux et hétérogènes, la méthodologie est insuffisante pour permettre de conclure.

Les revues de D. Benhamou, L. Condamine et S. Nicolian nuancent ce constat. Plusieurs essais thérapeutiques contrôlés récents bien menés avec l'acupuncture et les techniques corps-esprit attestent des effets bénéfiques sur certains types de douleurs et dans certaines indications. On les retrouvera détaillés dans les articles de cette livraison. Le niveau de conviction est tel que l'efficacité de l'acupuncture contre placebo est reconnue dans le dernier rapport de l'INSERM sur le sujet [5] : « Pour bon nombre de douleurs chroniques, pour traiter des nausées et vomissements, on peut affirmer avec suffisamment de certitude que l'acupuncture a une efficacité supérieure à une absence de soin... De ce fait, l'acupuncture pourrait offrir un complément intéressant dans le cadre d'une prise en charge plus globale de la maladie, en particulier lorsque la médecine traditionnelle n'est pas en mesure d'apporter un soulagement satisfaisant aux patients. » De son côté, l'organisme d'évaluation anglais NICE a reconnu à l'acupuncture l'indication « douleurs lombaires chroniques » [6].

Dès lors, quels bénéfices attendre d'une recherche clinique sur ces sujets ? L'intégration d'une médecine alternative et complémentaire dans un système de santé impose l'existence préalable d'une évaluation comparative et d'une régulation officielle [7]. Concernant l'évaluation comparative, deux problèmes se posent toujours : quel est le bon placebo de ces médecines très individualisées ? Quelle est l'efficience de ces procédures par rapport aux autres thérapeutiques, notamment médicamenteuses ? Quant à la régulation officielle, elle fait cruellement défaut dans notre pays. Trois avis récents peuvent illustrer ce propos. Concernant la douleur, la dernière mise au point de l'Agence Nationale de Sécurité du Médicament (ANSM) intitulée « Prise en charge des douleurs de l'adulte modérées à intenses » ne concerne que les thérapeutiques médicamenteuses et n'évoque pas la place respective des traitements médicamenteux par rapport aux autres méthodes, ni leur efficience [8]. La Haute Autorité de Santé a publié un « rapport d'orientation : développement de la prescription de thérapeutiques non médicamenteuses validées ». Les mécanismes et règles de cette validation ne sont pas connus [9]. Côté académique : on peut regretter que l'Académie de Médecine contribuât à diffuser des termes comme celui de "patamédecine » [10]. Quant aux recommandations de bonne pratique émanant de nos sociétés savantes, elles n'envisagent que très rarement l'efficacité et l'efficience des médecines alternatives et complé- mentaires. La France est de ce point de vue très isolée face aux autorités académiques ou aux régulations administratives de nombreux autres pays [2,6,11].

Pourtant, tous les sondages nationaux et étrangers rapportent des chiffres similaires. Au cours d'une maladie chronique, plus d'un patient sur deux a recours à une thérapie complémentaire, $90 \%$ de ces patients ont toujours confiance en leur médecin conventionnel. Mais beaucoup moins de la moitié lui « avouent » ce recours à une médecine non conventionnelle. Plus grave : le sujet est abondamment traité dans de nombreux journaux grand public. La couverture de ces tabloïds assimile souvent ces médecines complémentaires à des médecines sans médecin. Notre médecine dite factuelle et notre évaluation cartésienne contribueraient-elles à entretenir ces 400 thérapies recensées par la milivude et à développer un système de santé parallèle dans notre pays ?

\section{Conclusion}

En 2006, Antoine Lazarus, pionnier d'une réflexion intégrative, remettait au ministère de la Santé et des Solidarités un rapport d'étape sur les stratégies nouvelles de soins et prévention. Il concluait : «Il s'agit d'agir sur les déterminants environnementaux et sociaux et de permettre à chaque citoyen d'exercer un meilleur contrôle sur sa propre santé en créant les conditions qui lui permettent d'opter pour des choix en connaissance de cause. Le citoyen est le premier acteur de toute démarche de prévention » [12]. Cette stratégie d'écoute du citoyen, d'évaluation de sa demande, et d'information sur les médecines alternatives et complémentaires, la médecine chinoise notamment, s'applique parfaitement aux travaux de convergence de nos deux médecines.

\section{Références}

1. Zheng Z, Xue CC (2013) Pain Research in Complementary and Alternative Medicine in Australia: A Critical Review. J Altern Complement Med 19:81-91

2. Working Group Report (2015) A National Advisory Council on Complementary and Integrative Health (NACCIH) https://nccih. nih.gov/about/naccih/

3. Attali JR (2014) La médecine traditionnelle chinoise. pitiesalpetriere.aphp.fr/wp-content/blogs.dir/134/files/2014/04/ MTC-ATTALI.pdf

4. Zhu M (2004) La douleur dans la médecine traditionnelle chinoise. In: Journées d'étude APF Formation - Unesco - 21, 22 et 23 janvier 2004 http://apfformation.blogs.apf.asso.fr/ media/00/00/1947183820.pdf

5. Barry C, Seegers V, Gueguen J, et al (2014) Évaluation de l'efficacité et de la sécurité de l'acupuncture. www.inserm.fr/content/ download/82019/618732/file/Acupuncture

6. Savigny P, Watson P, Underwood M; Guideline Development Group (NICE) (2009) Early management of persistent non- 
specific low back pain: summary of NICE guidance. BMJ 338 : b1805

7. Fagon JY, Viens-Bitker C (2012) médecines complémentaires à l'Assistance Publique-Hôpitaux de Paris. Rapport. p 1-33

8. Groupe référent médecins généralistes de l'AFSSAPS (2011) Mise au point : prise en charge des douleurs de l'adulte modérées à intenses. http://ansm.sante.fr/var/ansm_site/storage/original/ application/a6497f74fc2f18e8db0022973f9327e1.pdf

9. Haute Autorité de Santé (2011) Rapport d'orientation : développement de la prescription de thérapeutiques non médicamenteuses validées. http://www.has-sante.fr/portail/upload/docs/application/ pdf/2011-06/developpement_de_la_prescription_de_therapeutiques non_medicamenteuses_rapport.pdf

10. Bontoux D, Couturier D, Menkes CJ (2013) Thérapies complémentaires -acupuncture, hypnose ostéopathie, tai-chi- leur place parmi les ressources de soins. Académie Nationale de Médecine. Rapport 5 mars 2013

11. Graz B, Rodondi PY, Bonvin E (2011) Existe-t-il des données scientifiques sur l'efficacité clinique des médecines complémentaires. Forum Med Suisse 11:808-13

12. Lazarus A, Delahaye G (2007) Médecines complémentaires et alternatives : une concurrence à l'assaut de la médecine de preuves? Les Tribunes de la santé 15:79-94 